\title{
Stakeholders Driven Requirements Engineering Approach for Data Warehouse Development
}

\author{
Manoj Kumar*, Anjana Gosain** and Yogesh Singh**
}

\begin{abstract}
Most of the data warehouse (DW) requirements engineering approaches have not distinguished the early requirements engineering phase from the late requirements engineering phase. There are very few approaches seen in the literature that explicitly model the early \& late requirements for a DW. In this paper, we propose an AGDI (Agent-Goal-Decision-Information) model to support the early and late requirements for the development of DWs. Here, the notion of agent refers to the stakeholders of the organization and the dependency among agents refers to the dependencies among stakeholders for fulfilling their organizational goals. The proposed AGDI model also supports three interrelated modeling activities namely, organization modeling, decision modeling and information modeling. Here, early requirements are modeled by performing organization modeling and decision modeling activities, whereas late requirements are modeled by performing information modeling activities. The proposed approach has been illustrated to capture the early and late requirements for the development of a university data warehouse exemplifying our model's ability of supporting its decisional goals by providing decisional information.
\end{abstract}

Keywords-Agent, Dependencies Among Agents, Stakeholders of the Organization, Data Warehouse Requirements Engineering, Early Requirements Engineering, Late Requirements Engineering

\section{INTRODUCTION}

A great interest has been shown in the development of Data Warehouses (DWs) as witnessed by reviewing the data warehousing literature of the last two decades. The initial thrust of DWs was in decision making that principally involved numerical facts and textual dimensions. In recent years, however, Data Warehouses have been proposed for domains of image data [1] and voice data [2]. For DWs development in the decision-making domain, two different approaches have been used, data-driven [3] and requirements-driven [4]. In the former, data is gathered from operational systems into DWs, whereas in the latter the attempt is to identify the information needs to be met by the DW. In these approaches, the real issue is that of DW design i.e. once the given data needs are understood what should be the logical structure of the DW? Proposals to add a conceptual layer on top of the basic data layer have been made, for example, by Jarke et al.

\footnotetext{
Manuscript received April 22, 2010; accepted June 25, 2010.

Corresponding Author: Manoj Kumar

* Department of Computer Science \& Engineering, Ambedkar Institute of Technology, Delhi, India (manojgaur@ yahoo.com)

** University School of Information Technology, Guru Gobind Singh Indraprastha University, Delhi, India (anjana_ gosain@hotmail.com, ys66@rediffmail.com)
} 
[5]. In that proposal, it is assumed that the conceptual objects can be readily determined but the question of what useful conceptual objects are for a DW and how they are to be determined is not addressed. To answer this, we need an explicit Requirements Engineering (RE) phase in DW development. The requirements engineering stage has been divided into two phases: the early requirements engineering phase and the late requirements engineering phase [6, 7]. The "earlyphase" of requirements engineering activities include those that consider how the intended system would meet organizational goals, why the system is needed, what alternatives might exist, what the implications of the alternatives are for various stakeholders, and how the stakeholders' interests and concerns might be addressed. The emphasis here is on understanding the 'whys' that underlies system requirements [8], rather than on the precise and detailed specification of "what" the system should do. The notion of agent and related mentalisic notions are used in all software development phases from early requirements analysis down to the actual implementation $[7,9,10]$. The mentalistic notions can be founded on BDI (Belief, Desire, and Intention) agent architecture [11].

The various Data Warehouse Requirements Engineering (DWRE) approaches have not distinguished the early requirements engineering phase from the late requirements engineering phase. However, in [12] the early phase discussed in [9] has been extended to the requirements engineering of data warehouses. This approach [12] uses stakeholder dependencies, which are represented in an actor diagram. Thereafter, two perspectives are proposed, organizational and decisional. In the former, facts are identified and associated with goals of different actors. In the later, each fact is related with their dimensions and a set of measures is found out and associated with facts. This goal driven approach [12] is mainly focused on DW conceptual design, which can be employed within both supply driven and mixed supply/demand driven design frameworks for DWs. However, this approach does not look at the decisional goals [13] of the organization for which the DW is to be built. In [13], the GDI (Goal-Decision-Information) model [14] has been viewed in two ways, one from the organizational and the other from the technical perspective. The former looks upon the warehouse as embedded in an organization and considers the manner in which it supports organizational tasks. The latter deals with issues of data warehouse contents and their broad properties etc. This approach [13] is modeling the late phase requirements but not the early phase requirements in the context of DWs.

In this paper, we propose an AGDI (Agent-Goal-Decision-Information) model for the early \& late requirements engineering of DWs by extending the GDI model proposed in [14]. Here, we introduce the notion of agent, which represent stakeholders of the organization for which the DW is to be built. The agent may be an internal agent or an external agent, the former represent the stakeholder internal to the organization whereas the later represent the stakeholder external to the organization. The stakeholders' objectives are captured through the notion of a goal. The stakeholders of the organization may fulfill their objectives themselves or may be dependent upon other stakeholders to fulfill their objectives. The stakeholders' dependencies have been modeled as goal, decision and information dependencies among agents and are shown through the 'delegate' relationship in figure 2 as discussed in section 4. The proposed AGDI model supports three interrelated modeling activities namely, organization modeling, goal modeling and decision modeling to capture the early and late requirements for a data warehouse as follows:

The organization modeling as in $[9,12]$ looks at the organizational context and identifies the agents and their associated goals for representing stakeholders and their objectives. The output of the organization modeling is represented as an organization model showing agents and their 
goal dependencies. This organization model is further refined until all the complex goals of an agent are converted into simple goals, which may be delegated to other agents. This refined organization model is given as input for the decision modeling. During decision modeling, the agent will suggest necessary decisions for achievement of the simple goals of all agents. The complex decision will further be refined into simple decisions through agent interaction. The output of decision modeling is represented as a decision model showing agents and their decision dependencies. The organization model captures various stakeholders and their objectives, whereas the decisions model captures how the stakeholders may fulfill their objectives. These organization and decision models represent the 'whys' that underlie a system's requirements, i.e. early requirements for a DW. The decision model is fed to the information modeling activity where a set of information supporting all the decisions is identified through agents' interaction. The output of the information modeling is represented as an information model showing agents and their information dependencies to support a particular decision. The set of information identified during information modeling may be kept in a data warehouse to support decision making activities in the organization. The information models capture the 'what' of a system's requirements, i.e. the late requirements for a data warehouse.

Organization of the paper is as follows: related literature is presented in section 2. Section 3 gives an overview of the GDI model. Section 4 discusses the proposed AGDI Model, which extends the GDI model to support requirements engineering issues for a DW. Section 5 discusses the early and late requirements modeling for a DW. The proposed approach is demonstrated in section 6 for requirements engineering of a university data warehouse, followed by our conclusions in section 7 .

\section{RELATED LITERATURE}

In recent years, a number of proposals for Requirements engineering of DW systems have been made. Efforts have also been made to define the Systems Development Life Cycle, SDLC, for DW development $[15,16]$. Both these assume the ER diagram as the input to their DW Requirements engineering phase. Some approaches assume that the main issue of DW development is to decide which process to improve and what improvements should be made. Bohnlein and Ulbrichvom Ende [17] present an approach that is based on the SOM (Semantic Object Model) process modeling technique. The DW's measures and dimensions are determined from an initial study of the goals and services to be provided by the organization and by subsequently carrying out an analysis of the processes adopted. Kimball [18] proposes a four-step approach where, from an initial choice of a business process, dimensions and facts are determined. He defines a business process as a major operational process in the organization that is supported by legacy systems. According to Frendi et al. [19], DW requirements can be elicited using business process requirements and strategic decision processes. They propose that DW models are produced using a combination of DW requirements and as-is data models. Once produced, DW data models can also be used to elicit new requirements. Winter [20] points out that a detailed analysis of business processes is not a good starting point for DW development. They propose a methodology to develop a DW system that exclusively supports decision processes. A different approach is adopted in [21] where the problem is to extract data marts [3] from the enterprise wide information system. It adopts the top-down approach for determining goals using the Goal- 
Question-Metric approach. The discovered goals are aggregated and refined in abstraction sheets from which ideal star schemata are extracted. It uses the bottom-up approach to look at ER schemata of operational databases and extracts candidate star schemata. The ideal star schemata are matched with these and candidate star schemata are ranked according to the metrics for selection. It can be seen that the information content of a DW is found in the larger context of the goals and objectives of an organization. These goals and objectives identify the decision-making capability to be supported. Therefore, it is proposed in [14] that the identification of goals suggests the decisions that influence the satisfaction of these goals. Implementation of decisions is done by actions associated with them. Finally, from knowledge of the decisions, it is possible to identify the information that is needed to make the decision. Here, the product of requirements engineering is represented as a schema of the GDI model [14]. The easy requirements modeling technique for a DW system [22] represents good practices as requirements management. This approach [22] focuses on the communication between the stakeholders and users of a new data DW system and those who are building it. Furthermore, it helps to apply an effective requirements engineering method by the use of different perspectives for representing DW requirements. Our proposed agent oriented approach mainly focuses on capturing stakeholders and their dependency for achieving their objectives. Our approach shares some similarities with the goal driven approach [12] and also with the easy requirements modeling techniques for a DW system [22]. The GDI model [14] is discussed in the next section that will form the basis for the proposed approach.

\section{GDI (GOAL-DECISION-INFORMATION) MODEL FOR DATA WAREHOUSE REQUIREMENTS ENGINEERING}

The goal-decision-information (GDI) model [14] is shown in Figure1. In accordance with goal-orientation [23, 24], a goal is viewed as an aim or objective that is to be met. A goal is a passive concept and unlike an activity/process/event it cannot perform or cause any action to be performed. A goal is set, and once so defined it needs an active component to realize it. The active component is a decision. Further, to fulfill the decisions the appropriate information is required. As shown in Figure1, a goal can be either simple or complex. A simple goal cannot be decomposed into simpler ones. A complex goal is built out of other goals which may themselves be simple or complex. This forms a goal hierarchy. The component goals of a complex one may be mandatory or optional. A decision is a specification of an active component that causes goal fulfillment. However, it is not the active component itself i.e. when a decision is selected for implementation then one or more actions may be performed to give effect to it. In other words, a decision is the intention to perform the actions that cause its implementation. Decision-making is an activity that results in the selection of the decision which is to be implemented. It is while performing this activity that information to select the right decision is needed. As shown in Figure1, a decision can be either simple or complex. A simple decision cannot be decomposed into simpler ones whereas a complex decision is built out of other simple or complex decisions. Figure1 shows that there is an association 'is influenced by' between goals and decisions. This association identifies the decisions which when taken can lead to goal satisfaction. The knowledge necessary to take decisions is captured in the notion of decisional information shown in Figure1. This information is a specification of the data that will eventually be stored in the Data Ware- 


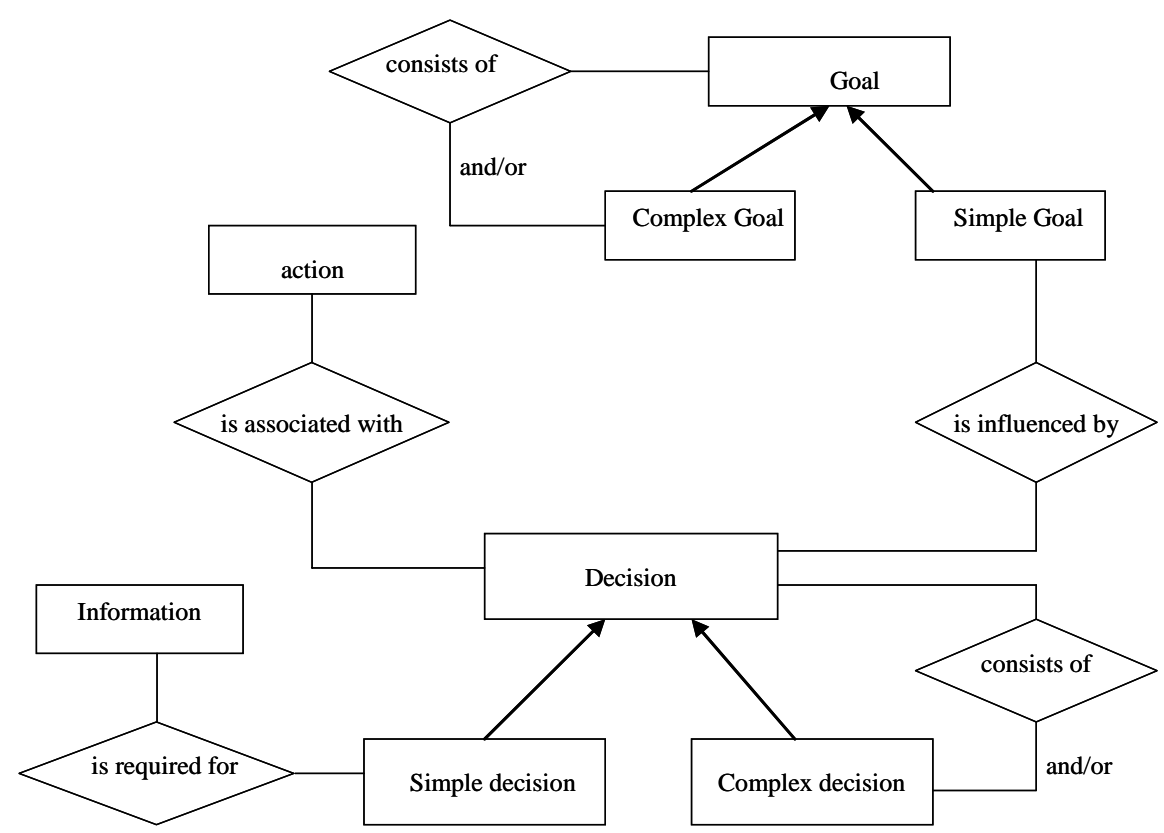

Fig. 1. GDI (Goal-Decision-Information) model for data warehouse requirements engineering

house. Figure1 shows that there is an association 'is required for' between decisions and decisional information. This association identifies the decisional information required to make a decision. This GDI model does not explicitly capture the stakeholders of the organization and their dependencies for achieving the goals, thus it is extended in section 4.

\section{AGDI (AgENT-GOAL-DECISION-INFORMATION) MODEL FOR DATA WAREHOUSE REQUIREMENTS ENGINEERING}

The GDI model [14] starts with the determination of the goals of the organization with the help of decision makers based on the assumption that only decision maker are the stakeholders responsible for decision making activities in the organization. However, the other stakeholders and their dependencies for achieving the goals of the organization are also important to be modeled. Because of this assumption, the GDI model lacks the ability to model stakeholders explicitly. In this paper, it is proposed to introduce the notion of agent to the existing GDI model [14] in order to represent the stakeholders of the organization. We may call the extended GDI model as an AGDI model as shown (within the dotted line frame) in figure 2. We also model stakeholders' dependencies through agent dependencies in the proposed AGDI Model. The Agent may depend on another agent for goals to be achieved, decisions to be suggested and information to be provided. These dependencies among agents are called goal, decision and information dependencies respectively. Our agent oriented approach uses the proposed AGDI model to support three interrelated modeling activities namely, organization modeling, goal modeling and decision modeling to capture the early and late requirements for a DW. Prior to discussing these 


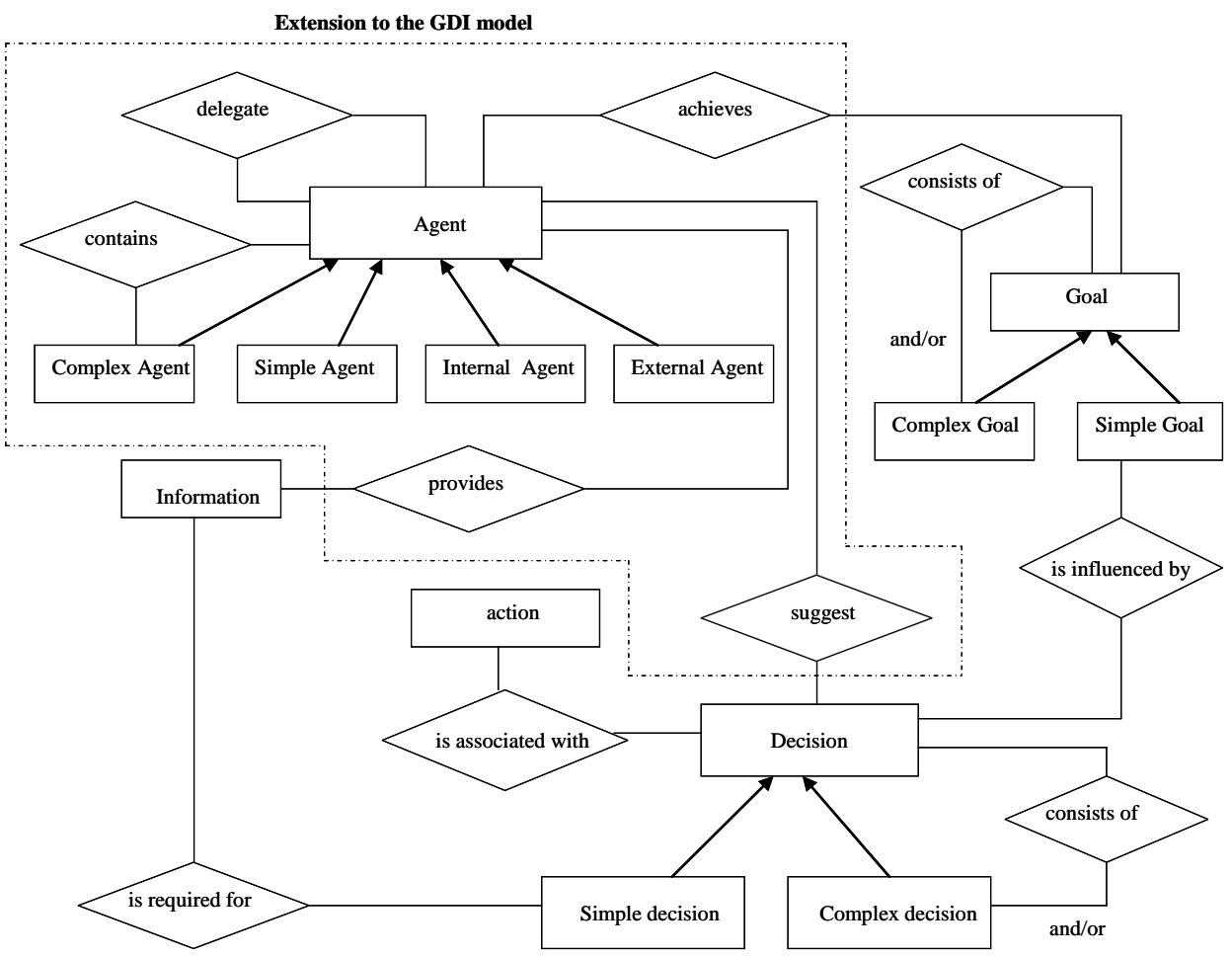

Fig. 2. AGDI (Agent-Goal-Decision-Information) model for requirements engineering of a data warehouse

modeling activities, we will discuss the various concepts used in the AGDI model of the proposed approach in the next sub section.

\subsection{Agent}

The concept of an agent can be traced back to the early days of research into Distributed Artificial Intelligence. Hewitt [25] proposed the concept of a self-contained, interactive and concurrently executing object, which he termed 'actor'. Here, an actor is a computational agent, which has a mail address and a behavior. Agents can be identified by seven types: collaborative agents, interface agents, mobile agents, information/internet agents, reactive agents, hybrid agents and smart Agents [26]. Another issue of note is that agents need not be benevolent to one another. It is quite possible that agents may be in competition with one another, or perhaps quite antagonistic towards each other. However, it has been viewed in [26] that it is possible to have competitive collaborative-type agents, competitive interface agents, and competitive information agents. Here, the notion of agent models various stakeholders playing different roles for the organization: decision makers, resource (information) provider, action implementer, etc. The agent may be an internal agent or an external agent as shown in figure 2. The internal agent models the internal stakeholders of the organization whereas the external agent models the external stakeholders of the organization as shown in figure 2. For example, in the case of a University Sys- 
tem, the student and the staff of the university may be internal agents whereas funding agencies and regulatory agencies may be the external agents. The external agents may provide various financial/informational resources to the organizational agents in order to control/regulate the decisional activities in the organization. The agent can either be a simple agent or a complex agent as shown in figure 2. The complex agent may contain a simple agent or complex agent, as shown through the 'contains' relationship in figure2. For example, the decision maker is a simple agent whereas the organization, for which the DW is to be built, is a complex agent. We may keep on decomposing the complex agent until we get simple agents who can actually contribute to achieve the goals of the organization.

\subsection{Agent \& Goal}

An agent may have goals to be achieved. Goals are valuable in identifying, organizing and justifying system requirements [23, 27]. In accordance with goal-orientation [17, 23], we view a goal as an aim or objective that is to be met. The goals have been classified in different ways [13, 27, 28, 29, 30, 31]. According to Van [27], goals are functional or non functional and the differentiation is made on the basis of the purpose behind the goal. According to Prakash [13], a facetattribute-value approach is adopted to develop a framework for goal classification. Prakash [13] pointed out that the purpose behind the goal may be decisional and termed the goal as a decisional goal. Here, we are focusing on decisional goals. An agent may achieve the goal on its own or an agent may be dependent upon another agent for achievement of the goal. The former case is shown through the 'achieves' relationship and the later case is shown through the 'delegate' relationship in figure 2. In the later case, one agent is delegating its goal to another agent and the receiver agent should be committed to achieve the goal. For example, the University as a complex agent may depend upon the internal agent 'Head of University' to achieve the goal 'Maintain Academic Excellence'.

\subsection{Agent, Decision \& Information}

A goal is set, and once so defined it needs an active component to realize it. The active component is a decision. The agent will be asked to suggest the relevant decisions for achievement of goal, which is shown through the 'suggest' relationship in figure2. For example, 'Dean' as an agent may have a goal 'Improve Lab facilities' and to achieve this goal, the agent may suggest various decisions such as: 'select lab for improvement 'and 'survey latest facilities'. An agent may provide the information required to support the decision, which is shown as the 'provides' relationship in figure2. An agent may also be dependent upon other agents for the required information to support the decision. For example, the agent 'HODs' may require the information 'course wise students' academic feedback' and 'course wise students' performance feedback' over the last three years in order to support the decision 'select course for improvement'. The identified information should be made available in the DW. The decision once taken as suggested by the agent should influence the goals towards achievement of the goal as shown through the 'influence' relationship in figure2. The decision once taken will be implemented through the set of actions to be carried out by an agent, which is shown as the 'associated' relationship in figure2. This AGDI model is used to carry out various modeling activities to capture the early and late requirements for a DW to support the decisional goals of an organization as discussed in the next section. 


\section{EARLy \& LATE REQUiRements Modeling for Data Warehouses USING AN AGDI MODEL}

During these modeling activities, the agent, goal, decision and information concepts of an AGDI model have been represented as bubble, rounded rectangle, rectangle and hexagon graphic symbols respectively. The organization and decisions modeling activities model early requirements, whereas information modeling activities model the late requirements for a DW as discussed in the next subsections.

\subsection{Organization Modeling}

The organization modeling as in $[9,12]$ looks at the organizational context and identifies the agents and their associated goals. An agent may achieve its own goals or may operate to achieve goals on behalf of some other agents. It may also collaborate with or delegate to other agents for a specific goal. The output of the organization modeling is represented as an organization model showing agents and their goal dependencies. This organization model is further refined until all the complex goals of an agent are converted into simple goals, which may be delegated to another agent. This refined organization model will be given as input to the decision modeling activity.

\subsection{Decision Modeling}

During decision modeling, the agents suggest necessary decisions for achievement of the simple goals of all agents. The relevant decisions may be a simple or complex decision. The complex decision will further be refined into simple decisions through agent interaction. The output of decisions modeling is represented as a decisions model showing agents and their decision dependencies. The organization model captures various stakeholders and their intentions whereas the decision model captures how the stakeholders may fulfill their objectives. These organization and decision models represent the 'whys' that underlie system requirements, i.e. the early requirements for a DW. The decision model is fed to the information modeling activity where a set of information to support all the decisions is identified through agents' interaction.

\subsection{Information Modeling}

During information modeling, agents identify the relevant information to support various decisions. The output of the information modeling is represented as an information model showing agents and their information dependencies to support a particular decision. The set of information identified during information modeling may be made available in the DW. The set of produced information models capture the 'what' of the DW system, i.e. late requirements for a data warehouse.

\section{Illustration of Our Proposed Agent Oriented Approach on a UNIVERSITY SYSTEM: A CASE STUDY}

Guru Gobind Singh Indraprastha University (GGSIPU) has been setup by the Govt. in consultation with the Regulatory Agency to impart technical education. The university is run under the 
supervision of a Vice-Chancellor as Head of University along with a Registrar, Deans of schools, faculty and staff as other functionaries. The university offers various technical programs in their various schools and wants to excel in the area of academics and research so as to produce good technical manpower. The university needs adequate funds from the funding agency to discharge its academic activities; here Govt. may be a funding agency. The regulatory agency regulates the university during academic activities through its regulations. Here, we are performing various modeling activities to capture the early and late requirements for a DW to support the decisional goals of the university through organization modeling, decision modeling and information modeling.

\subsection{Organization Modeling}

In the organization modeling, we identify the various stakeholders as internal/external agents and their objectives as goals of the agents, which can be shown in figure 3 . We may identify the University as a complex agent, which contains faculty, staff and the students as internal agents whereas the regulatory agency and funding agency are external to the university and may be termed as external agents. The university wants to achieve the goal 'maintain academic excellence', whereas the funding agency wants to implement its education policy in the university system as shown in figure 3 . The university needs adequate funds from the funding agency and consequently the university may delegate this goal to the funding agency for its achievement as shown in figure 3.When the agent/goal is complex then further refinement is required whereby agents and their goals are converted into simple agent/goal, which is shown in figure 4 . The 'University' being a complex agent contains the simple agent 'Head of University', who can achieve the goal on behalf of the university. The agent 'head of university' views the goal 'maintain academic excellence' as a complex goal. The agent breaks down this complex goal into sub goals: improve academic infrastructure, attract good faculty and staff and attract good students as shown in figure 4. The agent 'head of university' perceives that all these sub goals are mandatory to achieve a complex goal, as shown through the 'and' link in figure 4 . This refining activity will continue until the entirety of complex goals/sub goals are converted into simple goals or the goals/sub goals have been delegated to other agents for their fulfillment.

The goal 'improve T\&P of students' and 'offer good quality entrance exam' may be delegated to the agent 'Head T\&P' and 'Controller Examination' respectively for their achievement as

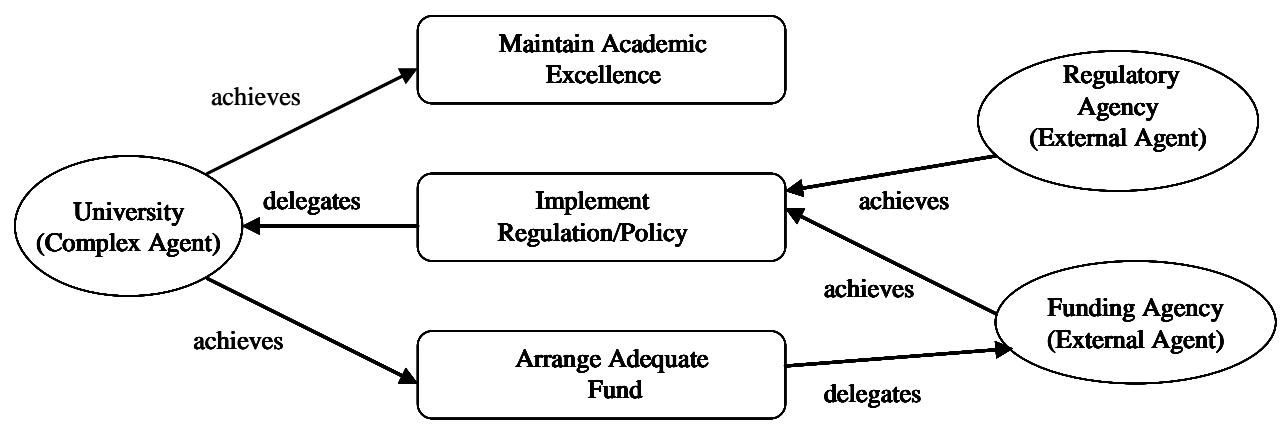

Fig. 3. Organization Model for a university - showing agents and their goal dependencies 


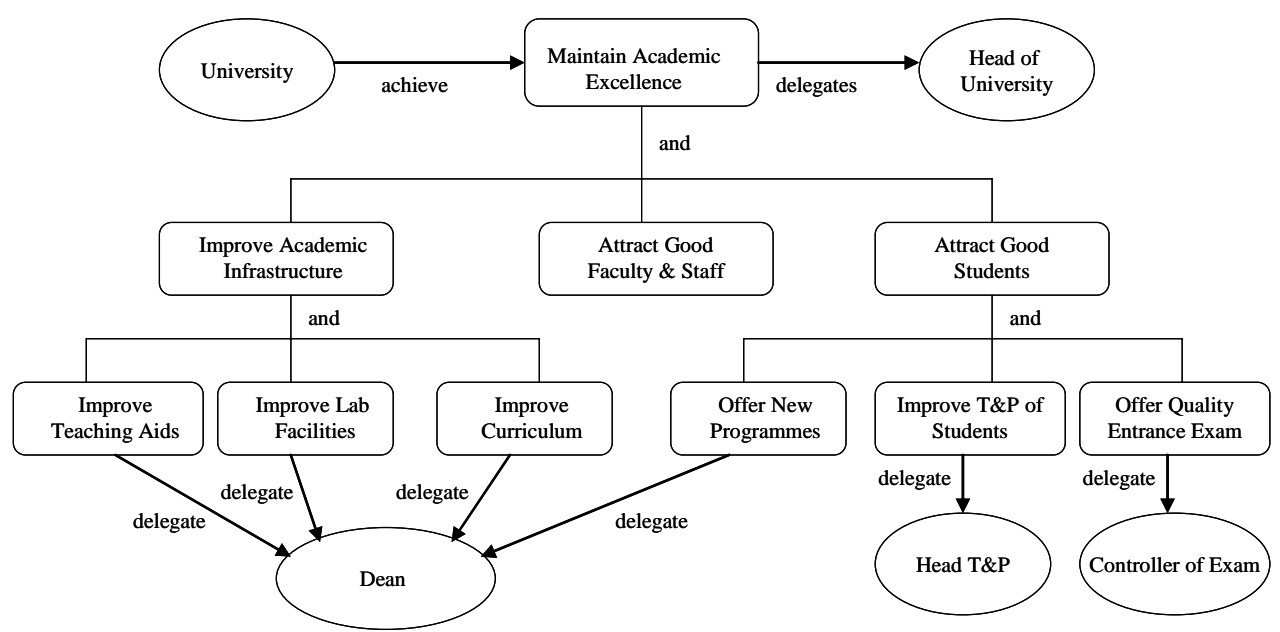

Fig. 4. Refined Organization Model for a university - showing new agents and their goal dependencies

shown through 'delegate' arrow links in figure 4. The refined organization model as shown in figure 4 will be given as input to the decision modeling, as discussed in the next section to follow.

\subsection{Decision Modeling}

During decision modeling, an agent will suggest the various decisions that may be taken to achieve the goal of the agent. The suggested decisions may be simple or complex decisions. The complex decisions are further refined into simple decisions. Keeping in view the refined organization model in figure 4 as input to the decision modeling, we can take 'Improve Curriculum' as a goal of an agent 'Dean'. In order to achieve this goal, the following decisions may be suggested by the agent 'Dean' as shown in figure 5:

(a) Select the course for improvement

(b) Constitute an expert committee

(c) Update course

(d) Approve course

The above-mentioned decisions (a), (c) and (d) are simple decisions and may be delegated to the agents namely, Heads of various departments (HODs), Expert Committee and Board of Studies (BOS) respectively for further fulfillment as shown in figure 5. The decision (b) is a complex decision, which is refined into two simple decisions namely, 'Select Industry Expert' and 'Select Academic Expert'. These two simple decisions are mandatory for achieving the complex decision as shown through the 'and' link in figure 5.

Similarly, for achieving a goal 'Improve Lab Facilities', 'Dean' as an agent may be suggesting the following decisions as shown in figure 6: 


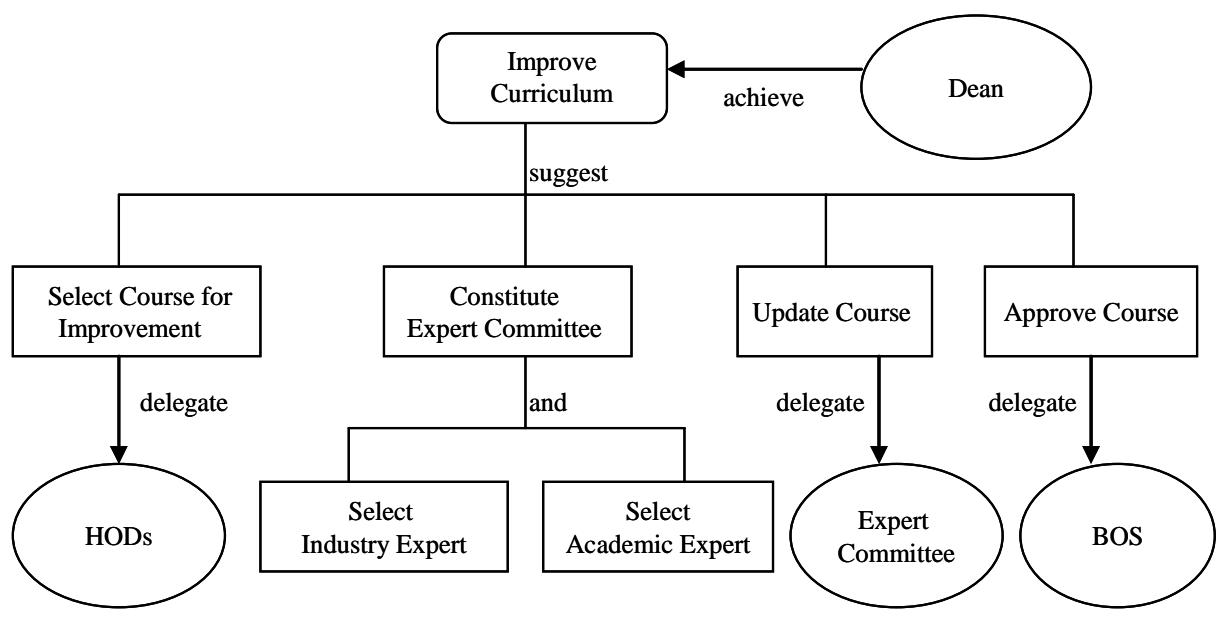

Fig. 5. Decision Model - showing agents and their decisions dependencies to achieve a goal 'Improve curriculum'

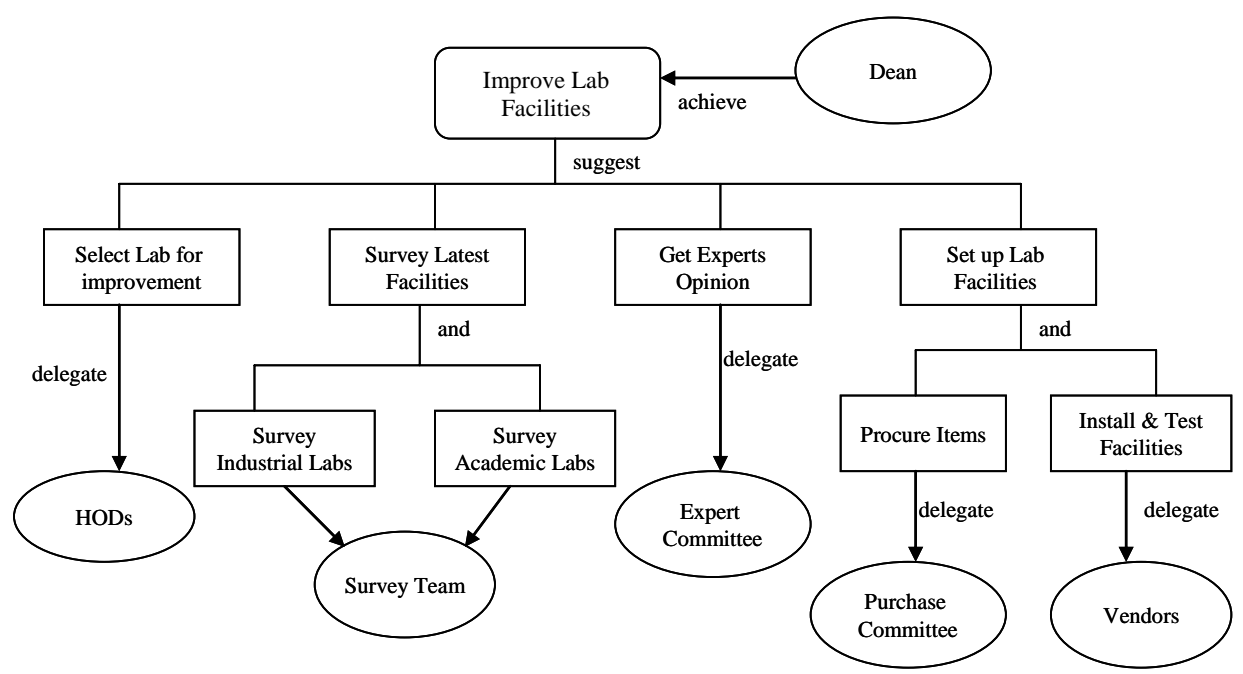

Fig. 6. Decision Model - showing agents and their decisions dependencies to achieve a goal 'Improve Lab facilities'

(a) Select lab

(b) Survey latest facilities.

(c) Get Experts Opinion

(d) Set up lab facilities

The decision (b) is a complex decision, which is built out of two simple decisions; 'Survey Industrial Labs' and 'Survey Academic Labs' and may be delegated to the agent 'Survey Team'. Similarly decision (d) is also a complex decision, which is built out of two simple decisions; 'Procure Items' and 'Install \& Test Facilities'. These two simple decisions may be delegated to the internal agent 'Purchase Committee' and the external agent 'Vendors' respectively. 
Similarly, for achieving a goal 'Improve Training and Placements (T\&P) of students', the following decisions may be suggested by the agent 'Head of Training \&Placement (T\&P)' as shown in figure 7 :

(a) Establish Industry-Institute Relationship

(b) Update Faculty \& Staff with latest technologies.

(c) Set up latest technical infrastructure

(d) Update curriculum as per industry requirements

The decision (b) is a complex decision, which is built out of two simple decisions; 'Sponsor faculty \& staff for advance study' and 'Encourage participation of faculty \&staff in international research programs'. These two simple decisions may be delegated to the agent 'Registrar' for achievement. The decision (c) may be delegated to the agent 'Head of Departments' (HODs) and decision (d) may be delegated to the agent 'Board of Studies' (BOS) for achievement.

Similarly, for achieving the goal 'offer good quality entrance exam', the following decisions may be suggested by the agent 'Controller of Exam' as shown in figure 8:

(a) Norms for selecting experts and also for conducting entrance exams.

(b) Set good quality question papers

(c) Review the standard of question papers

(d) Adopt latest technology for conducting the entrance exam

These decisions mentioned in (a), (b), (c) and (d) may be delegated to the agent 'Registrar', and to the external agents 'Experts', 'Review Committee' and 'Outsourcing Team' respectively for achievement. This decision modeling activity will be continued until all the goals of all the agents are taken into consideration. The output of decisions modeling is produced as a set of

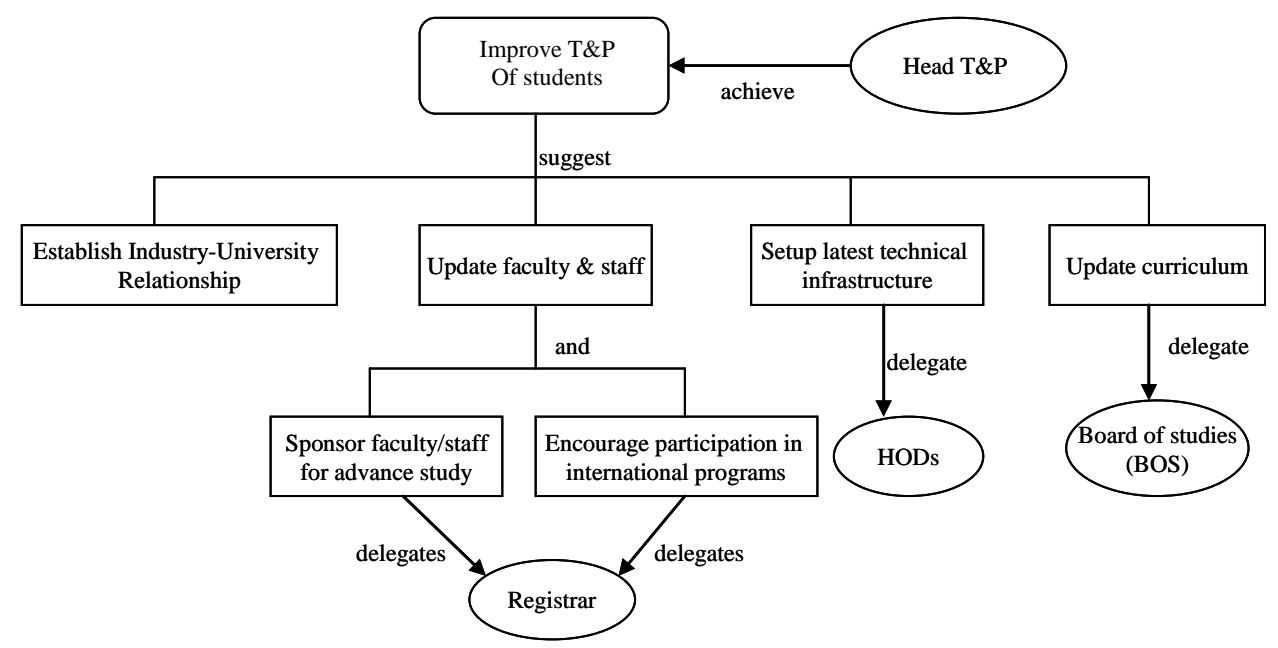

Fig. 7. Decision Model - showing agents and their decisions dependencies to achieve a goal 'Improve T\&P of students' 


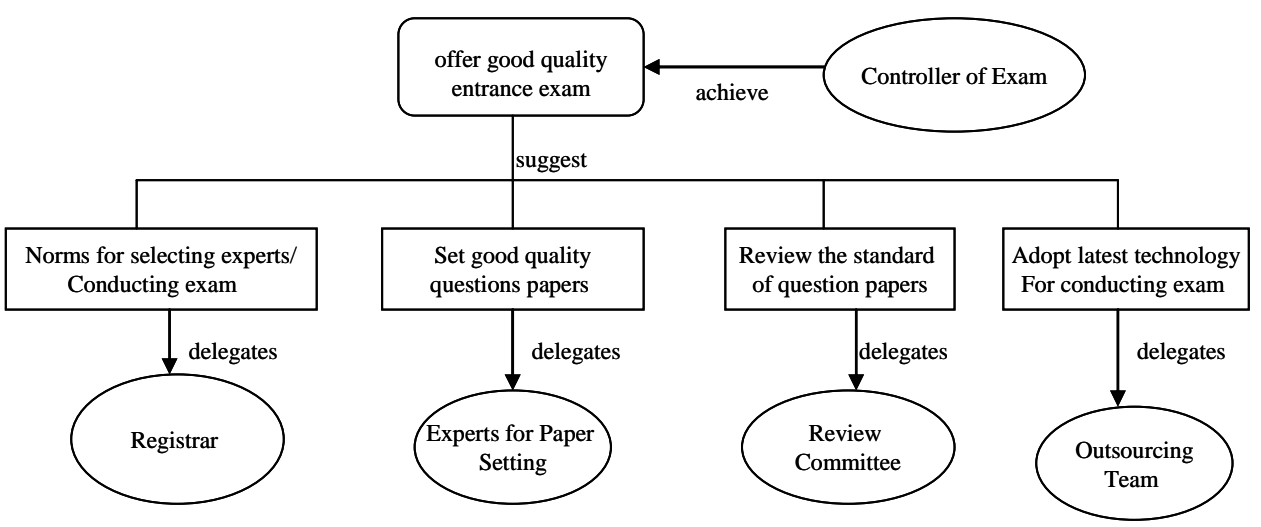

Fig. 8. Decision Model - showing agents and their decisions dependencies to achieve a goal 'offer good quality entrance exam'

decision models. These decisions models are fed as input to an information modeling activity in order to identify the required information to support the various decisions as suggested by the various agents. The information identification is carried out in the information modeling as discussed in the next section.

\subsection{Information Modeling}

During information modeling, the agents identify the needed information to support the various decisions. The information elicited during information modeling may be available in the DW to support the decisions. For example, to support the decision 'Select course for improvement' the following set of information may be required by the agent 'HODs' as shown in figure 9:

(a) Students' academic performance feed back for last years.

(b) Students' placements feedback for last years(course wise/year wise)

(c) Experts' comments regarding relevance, employability etc.

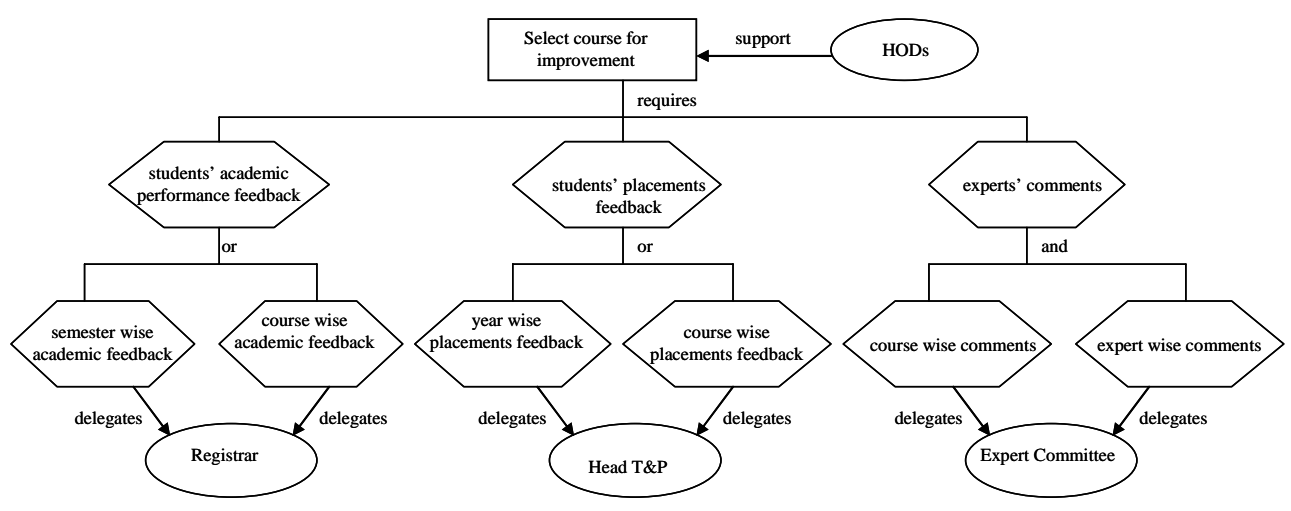

Fig. 9. Information model - showing agents and their information dependencies to support a decision 'select course for improvement' 
The information as mentioned in (a), (b) and (c) may be delegated to the various agents 'Registrar', 'Head of T\&P' and 'Expert Committee' respectively. These agents will actually provide the necessary information. The information as mentioned in (a) may either be needed as 'semester wise academic feedback' or 'course wise academic feedback' as shown by the 'or' link in figure 9. Similarly, the information mentioned as in (b) may either be needed as 'year wise placements feedback' or 'course wise placements feedback' as shown by the 'or' link in figure 9.

Similarly, the information as mentioned in (c) may be needed as 'course wise comments' and 'expert wise comments' as shown by the 'and' link in figure 9.

Similarly, for the decision 'select academic expert' the following information may be required by the agent 'Registrar' as shown in figure 10:

(a) List of internal academic experts with experience of teaching/revising the course (experience wise/ course wise).

(b) Norms for academic experts' selection

(c) List of external academic experts with experience of teaching/revising the course (experience wise/ course wise).

The information as mentioned in (a) may be delegated to the agent 'Joint Registrar' whereas the information as mentioned in (c) may be delegated to the external agent 'Other Universities'. These agents will help in providing the required information. The information as mentioned in (a) and (c) may be needed as either 'qualification/experience wise' or 'course wise' as shown by the 'or' link in figure 10. However, the information as mentioned in (b) may be available with the registrar itself, and thus not delegated to another agent.

Similarly, for supporting the decision 'approve course' the following information may be required by the agent 'Board of Studies' as shown in figure 11:

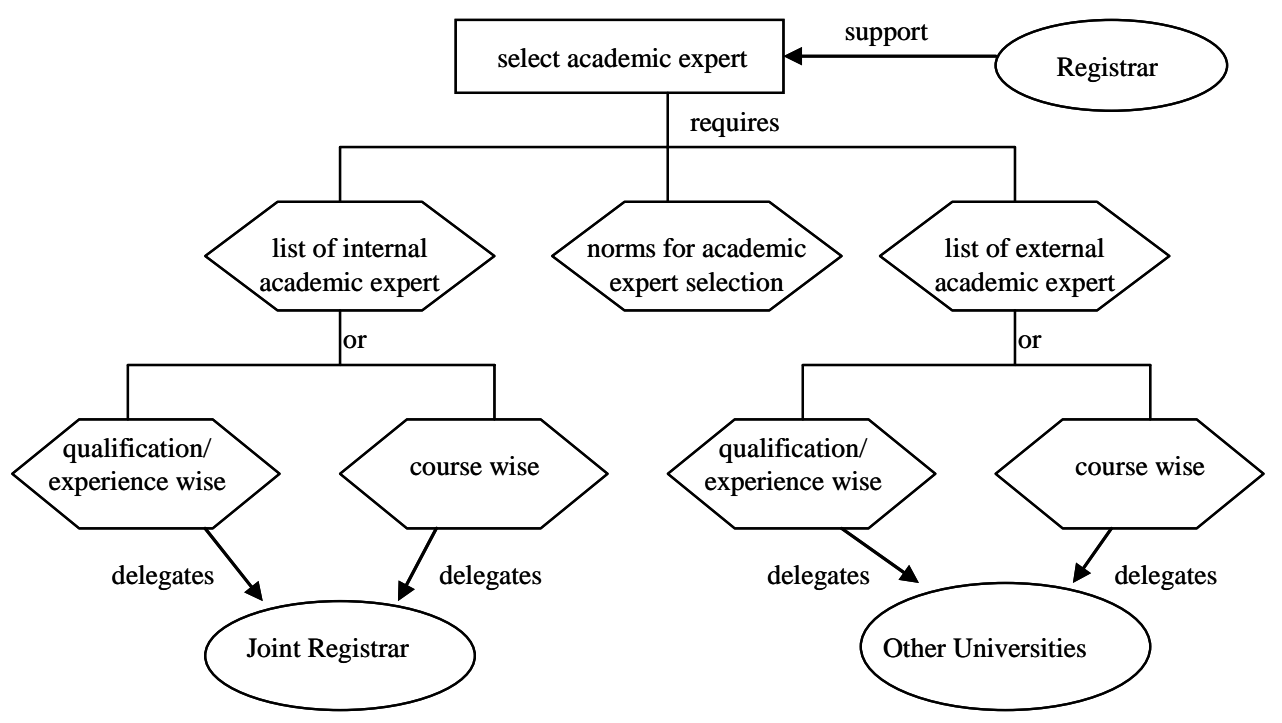

Fig. 10. Information model - showing agent and their information requirements to support a decision 


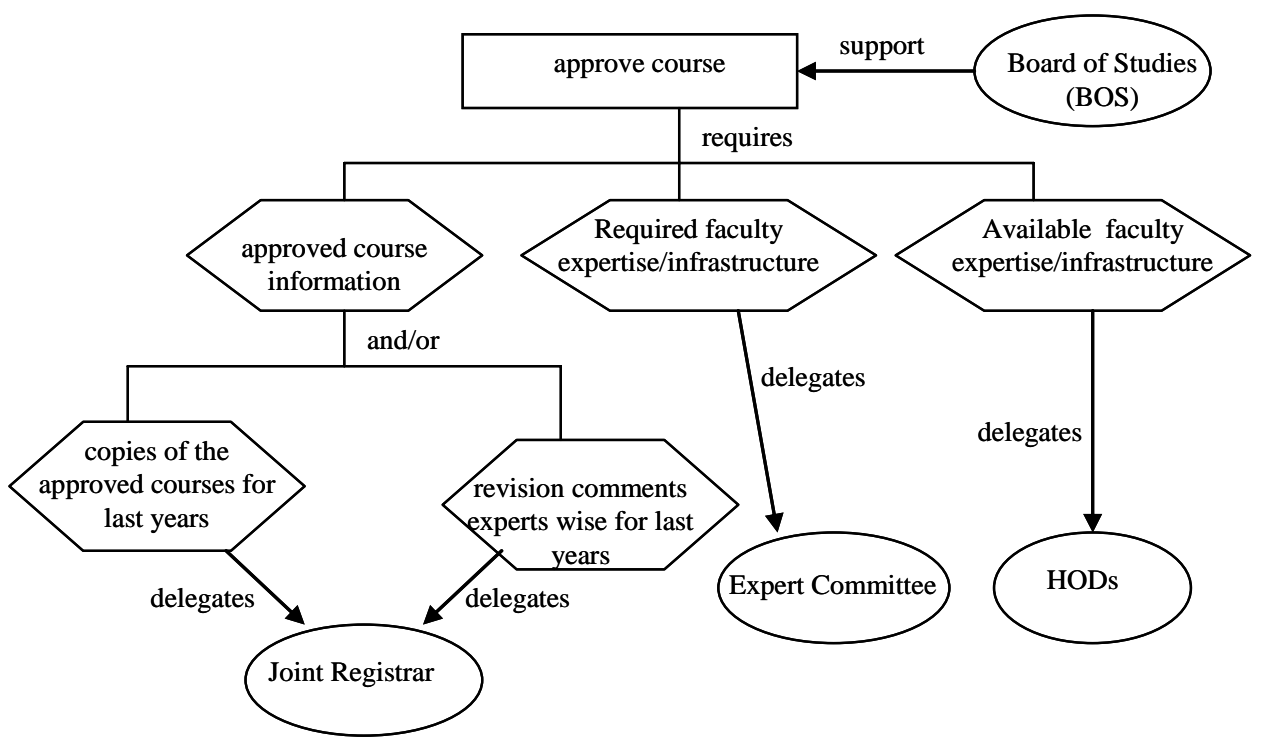

Fig. 11. Information model - showing agent and their information dependencies to support a decision 'approve coures'

(a) Copies of approved courses for last years with necessary comments of various members of the Board of Studies.

(b) Required faculty expertise/ infrastructure facilities in order to run the updated course.

(c) Availability of required faculty expertise/ infrastructure facilities in order to run the updated course.

The information as mentioned in (a), (b) and (c) may be delegated to the agents 'Joint Registrar', 'Expert Committee' and 'HODs' respectively for providing the required information. These agents will further provide the required information. The information modeling activity will be continued until the decisions of all the agents are taken into consideration. All the information identified during decision modeling should be available in the DW to support the different decisions that may be taken in the near future. The set of information models produced during information modeling activities capture the 'what' of the system requirements, i.e. the late requirements for a data warehouse.

\section{CONCLUSIONS \& FUTURE WORK}

The AGDI model has been proposed for the requirements engineering of data warehouses and used to capture the early and late requirements of a data warehouse from stakeholders' perspective. The early requirements have been modeled by producing an organization model and decision model, whereas the late requirements were modeled by producing an information model. Our proposed approach has been demonstrated for capturing requirements of a university data warehouse, as a case study. As a part of our future work, we will extend our proposed approach 
to address conceptual modeling issues for development of DWs and will also develop a CASE tool to support the modeling activities.

\section{REFERENCES}

[1] Wong, S.T., Hoo, K.S. Jr., Knowlton, R.C., Laxer, K.D., Cao, X., Hawkins, R.A., Dillon, W.P., Arenson, R.L., Design and applications of a multimodality image data warehouse framework, J. American Medical Information Association, 9, 239-254, 2002

[2] Miller, L.L., Honavar, V., Barta, T., Warehousing Structured and Unstructured Data for Data Mining, http://www.asis.org/annal-97/whouse.htm

[3] Inmon, W.H., Building the data warehouse, Wiley, New York, 1996.

[4] Ballard, C., Herreman, D., Schau, D., Bell, R.,Kim,E., Valencic, A., Data modeling techniques for data warehousing, redbooks.ibm.com

[5] Jarke, M., Jeusfeld, A., Quix, C., Vassiliadis P., Architecture and quality in data warehouses, proceedings of 10th CAiSE Conference, Springer, Heidelberg, 93-113, 1998.

[6] Yu, E.S.K., Modeling Strategic Relationships for Process Reengineering, Ph.D. thesis, Dept. of Computer Science, University of Toronto, Toronto, 1995.

[7] Yu, E.S.K., Towards Modeling and Reasoning Support for Early-Phase Requirements Engineering, proceedings of IEEE International Symposium on Requirements Engineering, 226-235, 1997.

[8] Yu, E., Mylopoulos, J., Understanding Why in Requirements Engineering with an Example, proceedings of Workshop on System Requirements: Analysis, Management, and Exploitation, 1994.

[9] Bresciani P., Giorgini P., Mylopoulous J., Perini A., TROPOS: An Agent-Oriented Software Development Methodology, J. Autonomous Agents and Multi-Agent Systems, 8, 203-236, 2004.

[10] Yu, E.S.K., Why Agent Oriented Requirements Engineering, proceedings of Third Workshop on Requirements Engineering, Barcelona, Catalonia, June 1997.

[11] Rao, A. S., George, M. P., Modeling rational agents within a BDI architecture, proceedings of Knowledge Representation and Reasoning Conference, 1991.

[12] Giorgini, P., Rizzi, S., Garzetti, M., GRAnD: A goal-oriented approach to requirement analysis in data warehouses, Elsevier Science Direct, Decision Support Systems, 45, 4-21, 2008.

[13] Prakash, N., Gosain, A., An approach to engineering the requirements of data warehouses, SpringerVeralag, Requirements Eng. Journal, 2007.

[14] Prakash, N., Gosain, A., Requirements driven data warehouse development, proceedings of CAiSE Short Paper, 13-17, 2003.

[15] Golfarelli, M., Rizzi, S., Designing the data warehouse: key steps and crucial issues, J. Computer Science Information Management, 2(3), 1999

[16] Husemann, B., Lechtenborger, J., Vossen, G., Conceptual data warehouse design, proceedings of the international workshop on design and management of data warehouses, Stockholm, Sweden, June 5-6, 2000.

[17] Boehnlein, M., Ulbrich vom Ende A., Deriving initial data warehouse structures from the conceptual data models of the underlying operational information systems, Proceedings of workshop on data warehousing and OLAP, Kansas City, MO, USA, 1991.

[18] Kimball, R., Reeves, L., Ross, M., Thornthwate, W., The data warehouse lifecycle toolkit. Wiley, New York, 1998.

[19] Frendi, M., Salinesi, C., Requirements engineering for data warehousing, proceedings of REFSQ Workshop, 2003.

[20] Winter, R., Strauch, B., Information requirements engineering for data warehouse systems, proceedings of ACM symposium on applied computing, Nicosia, Cyprus, 2003.

[21] Bonifati, A., Cattaneo, F., Ceri, S., Fuggetta, A., Paraboschi, S., Designing data marts for data warehouses. ACM Trans Software Eng Methodology, 10(4), 452-483, 2001.

[22] Shiefer, J., List, B., Bruckner, R.M., A holistic approach for managing requirements of data warehouse systems, proceedings of 8th Americas Conference on information systems, 2002. 
[23] Anton, A. I., Goal based requirements analysis, proceedings of the IEEE International Conference on Requirements Engineering, Colarado Springs, USA, 1996.

[24] Bubenko, J. A., Information Modeling in the Context of System De-velopment, proceedings of IFIP, 395-411, 1980.

[25] Hewitt, C., Viewing Control Structures as Patterns of Passing Messages, J. Artificial Intelligence, 8(3), 323-364, 1977.

[26] Hyacinth, S.N., Software Agents: An Overview, J. Knowledge Engineering Review, 11(3), 205-244, 1996.

[27] Van, L.A., Goal-oriented requirements engineering: a guided tour. Invited paper for RE’01, proceedings of 5th IEEE international symposium on requirements engineering, Toronto, August, 249-263, 2001.

[28] Sutcliffe, A., Maiden, N., Bridging the requirements gap: policies, goals and domains, proceedings of IEEE seventh international workshop on software specification and design, 1993.

[29] Rolland, C., Grosz, G., Kla, R., A proposal for a scenario classification framework, Requirement Engineering Journal, 1998.

[30] Mylopoulos, J., Chung, L., Nixon, B., Representing and using nonfunctional requirements: a processoriented approach, IEEE Trans Software Eng, 18(6), 483-497,1992.

[31] Dardenne, A., Van Lamsweerde, A., Fickas, S., Goal-Directed Requirements Acquisition, Science of Computer Programming, 20, 3-50, 1993.

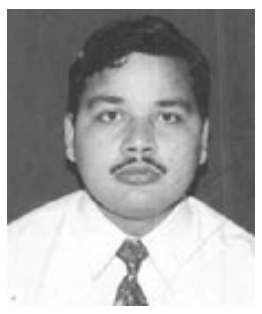

\section{Manoj Kumar}

$\mathrm{He}$ is working as Assistant Professor in the Department of Computer Science \& Engineering at Ambedkar Institute of Technology, Delhi. He has obtained his B.E. (CSE) from Kumaun University, Nainital in 1996 and M.Tech. in Information Systems from NSIT, University of Delhi in 2002. He has been pursuing a Ph.D from GGS Indraprastha University Delhi since 2007. Prior to joining the Ambedkar Institute of Technology, He had served as lecturer for 9 years at SLIET (Deemed to be a University), Longowal, Punjab. His technical and research interests include Data Warehousing, Requirements Engineering, Information Security, Software Engineering and Databases. He has published 10 research papers in International/National Journals and Conferences.

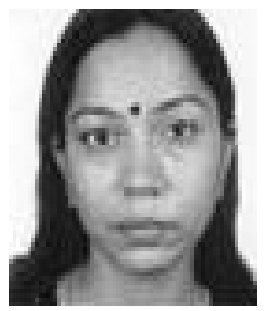

\section{Anjana Gosain}

She is working as reader at the University School of Information Technology. She obtained her Ph.D. from GGS Indraprastha University \& M.Tech in Information Systems from Netaji Subhas Institute of Technology (NSIT) Delhi. Prior to joining the school, she has worked with the computer science department of Y.M.C.A institute of Engineering, Faridabad (1994-2002). She has also worked with REC Kurukshetra. Her technical and research interests include data warehouses, requirements engineering, databases, software engineering, object orientation and conceptual modeling. She has published 18 research papers in International / National journals and conferences. 


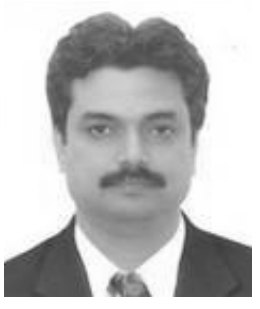

\section{Yogesh Singh}

$\mathrm{He}$ is a professor with the University School of Information Technology (USIT), Guru Gobind Singh Indraprastha University, India. He is also Controller of Examinations with the Guru Gobind Singh Indraprastha University, India. He was founder Head and dean of the University School of Information Technology, Guru Gobind Singh Indraprastha University. He received his master's degree and doctorate from the National Institute of Technology, Kurukshetra. His research interests include software engineering focusing on planning, testing, metrics, and neural networks. He is co-author of a book on software engineering, and is a Fellow of IETE and member of IEEE. He has more than 200 publications in International / National journals and conferences. 